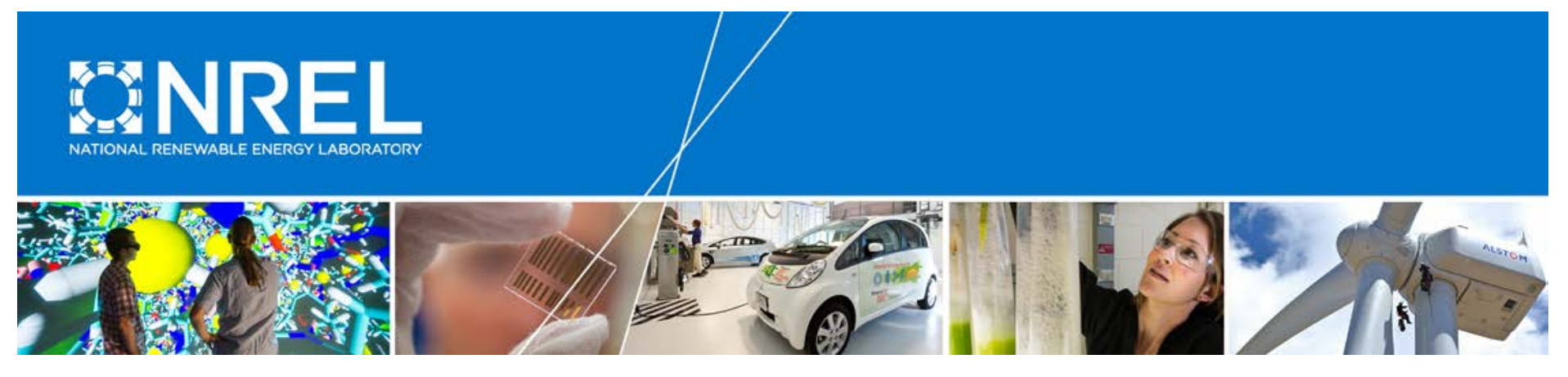

\title{
Automatic Regionalization Algorithm for Distributed State Estimation in Power Systems
}

\section{Preprint}

Dexin Wang and Liuqing Yang Colorado State University

Anthony Florita, S.M. Shafiul Alam, Tarek Elgindy, and Bri-Mathias Hodge National Renewable Energy Laboratory

To be presented at the IEEE Global Conference on Signal and Information Processing (GlobalSIP)

Washington, D.C.

December 7-9, 2016

(c) 2015 IEEE. Personal use of this material is permitted. Permission from IEEE must be obtained for all other uses, in any current or future media, including reprinting/republishing this material for advertising or promotional purposes, creating new collective works, for resale or redistribution to servers or lists, or reuse of any copyrighted component of this work in other works.

NREL is a national laboratory of the U.S. Department of Energy Office of Energy Efficiency \& Renewable Energy Operated by the Alliance for Sustainable Energy, LLC

This report is available at no cost from the National Renewable Energy Laboratory (NREL) at www.nrel.gov/publications.

\section{Conference Paper}

NREL/CP-5D00-66689

August 2016

Contract No. DE-AC36-08G028308 


\section{NOTICE}

The submitted manuscript has been offered by an employee of the Alliance for Sustainable Energy, LLC (Alliance), a contractor of the US Government under Contract No. DE-AC36-08GO28308. Accordingly, the US Government and Alliance retain a nonexclusive royalty-free license to publish or reproduce the published form of this contribution, or allow others to do so, for US Government purposes.

This report was prepared as an account of work sponsored by an agency of the United States government. Neither the United States government nor any agency thereof, nor any of their employees, makes any warranty, express or implied, or assumes any legal liability or responsibility for the accuracy, completeness, or usefulness of any information, apparatus, product, or process disclosed, or represents that its use would not infringe privately owned rights. Reference herein to any specific commercial product, process, or service by trade name, trademark, manufacturer, or otherwise does not necessarily constitute or imply its endorsement, recommendation, or favoring by the United States government or any agency thereof. The views and opinions of authors expressed herein do not necessarily state or reflect those of the United States government or any agency thereof.

This report is available at no cost from the National Renewable Energy Laboratory (NREL) at www.nrel.gov/publications.

Available electronically at SciTech Connect http:/www.osti.gov/scitech

Available for a processing fee to U.S. Department of Energy and its contractors, in paper, from:

U.S. Department of Energy

Office of Scientific and Technical Information

P.O. Box 62

Oak Ridge, TN 37831-0062

OSTI http://www.osti.gov

Phone: 865.576.8401

Fax: 865.576.5728

Email: reports@osti.gov

Available for sale to the public, in paper, from:

U.S. Department of Commerce

National Technical Information Service

5301 Shawnee Road

Alexandria, VA 22312

NTIS http://www.ntis.gov

Phone: 800.553 .6847 or 703.605 .6000

Fax: 703.605.6900

Email: orders@ntis.gov 


\title{
Automatic Regionalization Algorithm for Distributed State Estimation in Power Systems
}

\author{
Dexin Wang*, Liuqing Yang*, Anthony Florita ${ }^{\dagger}$, S M Shafiul Alam ${ }^{\dagger}$, Tarek Elgindy ${ }^{\dagger}$, and Bri-Mathias Hodge ${ }^{\dagger}$ \\ * Department of Electrical and Computer Engineering, Colorado State University, Fort Collins, CO 80523. \\ ${ }^{\dagger}$ National Renewable Energy Laboratory, Golden, CO 80401
}

\begin{abstract}
The deregulation of the power system and the incorporation of generation from renewable energy sources recessitates faster state estimation in the smart grid. Distributed state estimation (DSE) has become a promising and scalable solution to this urgent demand. In this paper, we investigate the regionalization algorithms for the power system, a necessary step before distributed state estimation can be performed. To the best of the authors' knowledge, this is the first investigation on automatic regionalization (AR). We propose three spectral clustering based AR algorithms. Simulations show that our proposed algorithms outperform the two investigated manual regionalization cases. With the help of $A R$ algorithms, we also show how the number of regions impacts the accuracy and convergence speed of the DSE and conclude that the number of regions needs to be chosen carefully to improve the convergence speed of DSEs.
\end{abstract}

Index Terms-automatic regionalization, distributed state estimation, partitioning, power system operations, spectral clustering

\section{INTRODUCTION}

State estimation is an essential tool for power system monitoring and analysis. Its results, the bus voltage magnitudes and phase angles, are critical inputs for other operational tools, such as contingency analysis, optimal power flow, and economic dispatch $[1,2]$. Traditionally, the state estimation algorithm takes the supervisory control and data acquisition (SCADA) measurements from substations and power plants as inputs and solves a weighted least squares (WLS) problem at a central processor. With the emergence and rapid development of power generation from renewable energy sources, the faster dynamics and the higher uncertainty of the availability of these energy sources will require that state estimation is performed more frequently. The time interval between consecutive state estimation updates needs to be dramatically reduced from 24 minutes to about 1 second. A single central processor is not capable of handling the tremendous computational load required by such state estimation tasks. Besides the aspect of processing power shortage, the communication load also increases due to the higher frequency of state estimation. With the installation of phasor measurement units (PMUs) in the smart grid, measurement data are becoming available at a much higher rate. A centralized structure would impose enormous load on the communication network and delays are inevitable.

Distributed state estimation (DSE) has attracted significant attention as a promising solution to this challenge (see e.g. [36]). In DSE, the entire system is first partitioned into smaller regions. This process is call regionalization. It is a necessary step before DSE can take place. With DSE, the computational load is distributed to multiple regional processors. The regional processors cooperate to estimate the state of the system. Each of them has access to local measurements within the region. In the literature, there are two types of DSE algorithms. In one type of DSE algorithms, each regional processor maintains a copy of the state vector of the entire system. In the other type of DSE algorithms, each regional processor only maintains a copy of the state vector of local buses. Both of the two types of DSE algorithms require some information exchange between neighboring regional processors to achieve a consensus among all processors. From our investigation, we observe that regionalization plays a crucial role in the performance of the DSE algorithms. However, in all the existing work on DSE, regionalization is performed manually and the DSE performance is typically evaluated only with a single arbitrary regionalization instance.

Furthermore, manual regionalization is inefficient and tedious for large systems. The main motivation for DSE is to improve the scalability of the state estimation algorithms to cope with large systems with hundreds, or even thousands of buses. In such systems, the number of regions (regional processors) also needs to be large in order to exploit more computational power, so that the processing time remains acceptable. Hence, manual regionalization becomes difficult and insufficient, if not impossible.

Therefore, in this paper, we investigate automatic regionalization (AR) for DSE. Inspired by [7], in which spectral clustering is employed for geographic regionalization, we propose to apply spectral clustering to power system regionalization. Our contributions include: 1) To the best of the authors' knowledge, this is the first investgation on automatic regionalization algorithms for power system DSE. 2) We propose three regionalization algorithms based on spectral clustering with different similarity measures. 3) We investigate the impact of the number of regions on the DSE accuracy and convergence speed through simulations.

The remainder of this paper is organized as follows. We first introduce spectral clustering in Section II. Then we describe our proposed regionalization algorithms in Section III. Simulations are presented in Section IV to evaluate the performance of our proposed algorithms, as well as to investigate the impact of the number of regions. Finally, Section V concludes the paper. 


\section{Spectral Clustering}

In this section, we introduce spectral clustering as background information for what follows in this paper. Clustering is an unsupervised machine learning technique aiming to group a set of objects so that objects in the same group are more similar to each other than to the objects in other groups. Compared with traditional clustering algorithms, such as $k$-means and hierarchical clustering algorithms, spectral clustering is a relatively new type of clustering algorithms. It is widely used in data analysis because of its often superior performance and the simplicity of implementation.

Spectral clustering is based on the similarity graph of the data points to be clustered. In this paper, the data points are the buses in the power system. Let $G=(V, E)$ be the undirected similarity graph, with vertex set $V=\left\{v_{1}, \cdots, v_{N}\right\}$. Each vertex represents a bus. The non-negative weight $w_{i j}$ of the edge $(i, j) \in E$ denotes the similarity between two data points. The definition of similarity is a vital factor to the performance of spectral clustering. In this application of spectral clustering to the regionalization problem, the definition of the similarity between buses will be discussed in detail in Section III. Let $\boldsymbol{W}=\left(w_{i j}\right)_{i, j=1, \cdots, N}$ be the weighted adjacency matrix of the graph $G$. The degree of vertex $v_{i}$ is defined as

$$
d_{i}=\sum_{j=1}^{N} w_{i j}
$$

and the degree matrix $\boldsymbol{D}$ is defined as the diagonal matrix with $d_{1}, \cdots, d_{N}$ on the diagonal.

The graph Laplacian matrix is

$$
\boldsymbol{L}=\boldsymbol{D}-\boldsymbol{W} .
$$

In this paper, we employ the normalized spectral clustering proposed in [8]. Let $\lambda_{1}, \cdots, \lambda_{k}$ be the $k$ smallest eigenvalues of the generalized eigenproblem $\boldsymbol{L} \boldsymbol{u}=\lambda \boldsymbol{D} \boldsymbol{u}$ and $\boldsymbol{u}_{1}, \cdots, \boldsymbol{u}_{k}$ be the corresponding eigenvectors. We denote $\boldsymbol{U}=\left[\boldsymbol{u}_{1}, \cdots, \boldsymbol{u}_{k}\right]$ as a $N \times k$ matrix.

Then, let $\boldsymbol{y}_{i} \in \mathbb{R}^{k}$ denote $i$ th row vector of $\boldsymbol{U}$, and with the $k$-means algorithm, we cluster $\boldsymbol{y}_{1}, \cdots, \boldsymbol{y}_{N}$ into $k$ clusters. Finally, from the clustering result of $\boldsymbol{y}_{i}$, we obtain the spectral clustering result of the corresponding vertex $v_{i}$. For a more detailed overview on spectral clustering, please refer to [9].

\section{Proposed Regionalization Algorithms}

In this paper, we investigate the problem of regionalization for DSE. In all existing DSE algorithms, the consensus among regions is achieved by each regional processor through adjusting the local state estimates according to information communicated from neighboring regions. Obviously, the DSE algorithm converges faster if the regions are more isolated. Therefore, the objective of our proposed automatic regionalization algorithms is to partition the entire system into a specified number of regions while minimizing the inter-region impact during the DSE process. In the following, we focus on two aspects of the algorithm design that help achieve this objective.

\section{A. Similarity Graph}

The construction of the similarity graph is an important factor to achieve a regionalization with minimum inter-region interactions during the DSE. It is desirable to have a definition of the similarity between a pair of buses that describes the mutual impact of the states of the buses, because buses with larger similarities tends to be clustered into one region in spectral clustering. Therefore, we propose the following three definitions of similarity.

1) Topology Based Similarity (TBS): A straightforward way to define similarity is to use the topology of the system directly. This results in a weighted adjacency matrix with 0 s and 1s. The weight of the edge between Bus $i$ and Bus $j$ is defined as

$$
w_{i j}= \begin{cases}1, & \text { A transmission line connects the buses, } \\ 0, & \text { Otherwise }\end{cases}
$$

2) Measurement Based Similarity (MBS): The states of a bus could impact its neighbor during the DSE only if there is an available measurement involving both buses. More specifically, a transmission line is actively involved in the DSE if, and only if, at least one power flow measurement is available on it, or one power injection measurement is available at one of the buses it connects. To capture this observation, the similarity can be defined as

$w_{i j}= \begin{cases}1, & \text { A measurement involving the buses is available, } \\ 0, & \text { Otherwise. }\end{cases}$

3) Weighted Measurement Based Similarity (WMBS): MBS considers the availability of the measurements. However, not every pair of buses are involved in the same number of measurements, and not all measurements have the same effect in coupling the involved buses. In some cases, it would be beneficial to divide two weakly coupled buses into different regions so that two strongly coupled buses can be clustered together. Therefore, the similarity between two buses is defined as the sum of the coupling strength metrics $c_{p}$ of all measurements involving both buses

$$
w_{i j}= \begin{cases}\sum_{p \in \mathcal{P}} c_{p}, & \mathcal{P}=\left\{p \mid \frac{\partial z_{p}}{\partial x_{i}} \neq 0 \& \frac{\partial z_{p}}{\partial x_{j}} \neq 0\right\} \neq \emptyset \\ 0, & \text { Otherwise. }\end{cases}
$$

For AC state estimation, $c_{p}$ is defined as a constant number 1 for all measurements. For DC state estimation, in which the relationship between measurements and states is linear, it is possible to define a better metric for the coupling strength of a measurement. Consider a measurement $z_{p}$ with the following relationship with the states

$$
z_{p}=\boldsymbol{h}_{p}^{T} \boldsymbol{x}+n_{p}
$$

where $\boldsymbol{x}$ is the state vector and $n_{p}$ is the measurement noise. The metric of its coupling strength is defined as $c_{p}=\left|\boldsymbol{h}_{p}\right|^{T} \mathbf{1}$, where $\left|\boldsymbol{h}_{p}\right|$ takes the element-wise absolute value of the vector $\boldsymbol{h}_{p}$ and $\mathbf{1}$ is the vector with all entries equal to 1 . The intuition behind this metric definition is that a measurement couples the buses more strongly if the states of involved buses have a larger influence on the measurement. This metric is not 
applicable to AC state estimation since the partial derivatives of the measurement change with the value of the states.

\section{B. Initialization of the $k$-Means Algorithm}

Note that the $k$-means problem is non-convex and NP-hard. Therefore, the algorithm is not guaranteed to find the global optimum. This means that the outcome of the algorithm is sensitive to the initial centroids selected for the algorithm. Hence, a good initialization method is necessary for the regionalization algorithm to perform well. Among the available initialization methods for the $k$-means algorithm, $k++$-means algorithm attempts to choose the $k$ initial centroids at locations evenly distributed in the sample space. This approach suits our purpose of regionalization well and, therefore, is chosen as the initialization method in our algorithm.

\section{Simulations}

We test our proposed regionalization algorithms on the IEEE 14-bus and 30-bus systems with the DC DSE algorithm proposed in [4]. The topology of the systems are shown in Fig. 1 and Fig. 2, respectively. The metric we use to evaluate the performance of regionalization schemes is the mean square error (MSE) of the state estimates and the number of iterations needed to converge within the tolerance of $10^{-3}$. In all the simulations, the measurement noise is assumed to be i.i.d. Gaussian with variance 0.1. We run 1000 Monte Carlo simulations for each scenario.

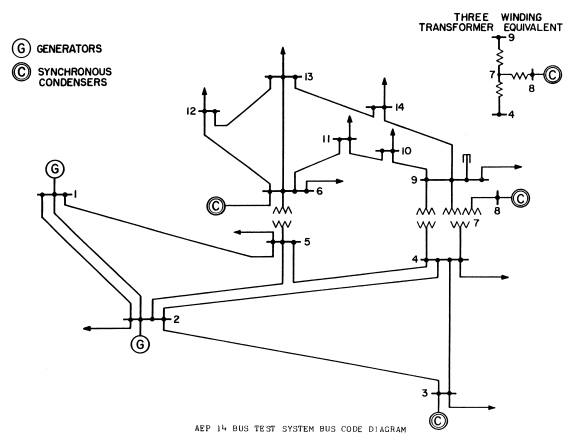

Fig. 1: IEEE 14-Bus System [10]

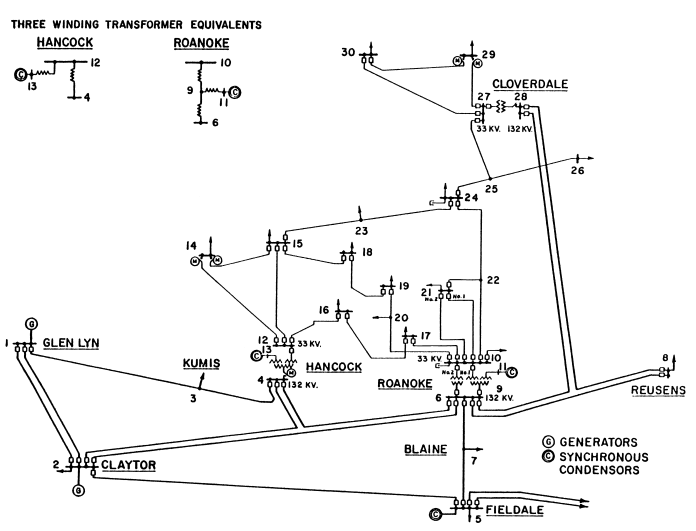

Fig. 2: IEEE 30-Bus System [10]

\section{A. Automatic vs. Manual Regionalization}

In this subsection, we compare the DSE performance under different regionalization schemes with 4 regions on the IEEE 14-bus system. Table I shows the regionalization scheme of the two manual cases, as well as the results of the three proposed AR algorithms.

TABLE I: Regionalization Schemes

\begin{tabular}{|c|c|c|c|c|}
\hline Regionalization & Region 1 & Region 2 & Region 3 & Region 4 \\
\hline Case 1 & $1,2,5$ & $3,4,7,8,9$ & $10,11,14$ & $6,12,13$ \\
\hline Case 2 & $1,2,5$ & $3,4,7,8$ & $9,10,14$ & $6,11,12,13$ \\
\hline TBS-AR & $1,2,3,4,5$ & $7,8,9$ & 10,11 & $6,12,13,14$ \\
\hline MBS-AR & $1,2,3,5$ & $4,7,8,9$ & 10,11 & $6,12,13,14$ \\
\hline WMBS-AR & $1,2,3,5$ & $4,7,8,9,14$ & 10,11 & $6,12,13$ \\
\hline
\end{tabular}

The MSE and the average number of iterations are presented in Table II. We observe from the data that the MSE of all regionalization algorithms are very similar. In terms of the speed of convergence, the regionalization schemes obtained by MBS-AR and WMBS-AR enable the DSE algorithm to converge with many fewer iterations at the same tolerance setting, especially for WMBS-AR. The TBS-AR, however, yields very slow convergence speed compared with the other two AR algorithms. The fact that TBS-AR only considers the topology of the system, instead of the availability of measurements, is the main reason for its poor performance.

TABLE II: SE Performance Comparisons

\begin{tabular}{|c|c|c|}
\hline & MSE & Average No. of Iter. \\
\hline DSE Case 1 & 0.0465 & 14035 \\
\hline DSE Case 2 & 0.0464 & 29889 \\
\hline TBS-AR & 0.0476 & 32538 \\
\hline MBS-AR & 0.0432 & 12299 \\
\hline WMBS-AR & 0.0446 & 12222 \\
\hline
\end{tabular}

To further investigate the cause of performance differences yielded from the three proposed AR algorithms, we focus on the discrepancies among their resultant regionalization schemes. In Table I, the discrepancy between regionalization scheme by TBS-AR and those by the other two AR algorithms lies in the affiliation of Bus 4 . Bus 4 was partitioned with Buses 1, 2, 3, and 5 by TBS-AR to avoid the inter-region connections between Bus 4 and Buses 2, 3, 5, respectively. However, both MBS-AR and WMBS-AR ignored this point and partitioned Bus 4 with Buses 7, 8, and 9. This is because in this simulation, the transmission line between Bus 2 and Bus 4 was not actively involved in any available measurement. MBS$\mathrm{AR}$ and WMBS-AR recognized its inactivity and ignored the transmission line during regionalization. This helps reducing the overall inter-region connections that are actually active. Comparing the resultant regionalization schemes of MBS-AR and WMBS-AR, the discrepancy occurred at Bus 14. WMBS$\mathrm{AR}$ is able to discern that Bus 14 has a stronger tie with Bus 9 than it has with Bus 13, whereas MBS-AR cannot. Therefore, with the regionalization scheme of WMBS-AR, the more strongly coupled buses are effectively partitioned into same regions. This is the main reason for its superior performance.

\section{B. Number of Regions}

The number of regions allowed has a significant impact on the performance of DSE, especially on the convergence speed. 
In this set of simulations, we investigate its impact on the performance of DSE with AR algorithms.

For the 14-bus system, the MSE and the average number of iterations are presented in Fig. 3. As shown in Fig. 3a, the basic trend is that the MSE increases slightly with the number of regions. In Fig. 3b, we can see that the number of iterations also increases with the number of regions. Therefore, when partitioning the system into more regions, the tradeoff between the ability to exploit more processing power and the cost of slower convergence, in terms of the number of iterations, has to be considered in system design.

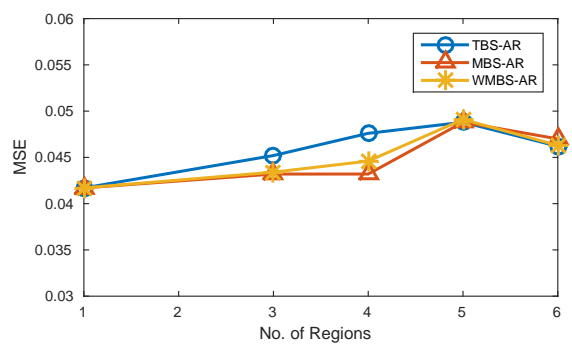

(a) MSE

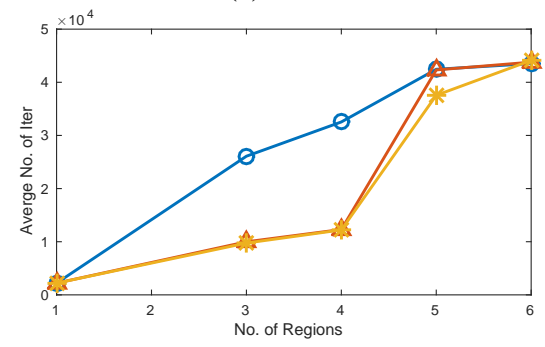

(b) Average Number of Iterations

Fig. 3: Impact of the number of regions on the IEEE 14-bus system.

For the 30-bus system, whose results are shown in Fig. 4, both the MSE and the average number of iterations needed for convergence is unusually high with 3 regions. And the convergence speed for MBS-AR and WMBS-AR is dramatically faster when partitioning the system into 4 regions. These somewhat counter-intuitive results can be explained as follows. The topology of the 30-bus system and the selection of measurements in the simulations result in a scenario more suitable to be partitioned into 4 regions than 3,5, or 6 regions.

Another interesting phenomenon worth noting is that the numbers of iterations needed by MBS-AR and WMBS-AR are much smaller than that by TBS-AR with 3 or 4 regions in the 14-bus system and then suddenly increase to a similar level with TBS-AR when the system is partitioned into 5 regions. For a small system such as the 14-bus system, as the number of regions increase, the probability of obtaining singleton regions are higher. Therefore, different AR algorithms tends to yield similar results with the average number of buses in each region is close to 1 .

\section{CONCLuSions}

In this paper, we investigated automatic regionalization algorithms for DSE in the smart grid. We first proposed three automatic regionalization algorithms based on spectral

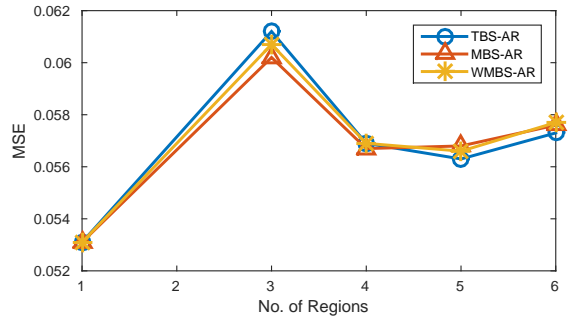

(a) MSE

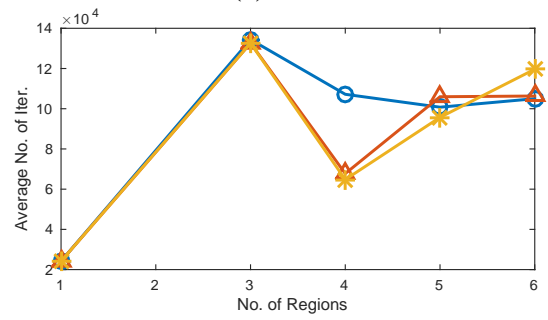

(b) Average Number of Iterations

Fig. 4: Impact of the number of regions on the IEEE 30-bus system.

clustering. Then we evaluated their performance through simulations. Compared with two manual regionalization cases, simulations show that our proposed AR algorithms provide better regionalization results that facilitate the DSE algorithm to achieve faster convergence. Additionally, we investigated the impact of the number of regions on DSE performance in terms of accuracy and convergence speed. Simulations show the number of regions significantly impacts the convergence speed in terms of the needed number of iterations. Therefore, the number of regions needs to be chosen carefully to achieve fast convergence.

\section{REFERENCES}

[1] F. F. W. D.M. Falcao and L. Murphy, "Parallel and Distributed State Estimation," IEEE Trans. Power Systems, vol. 10, no. 2, pp. 724-730, 1995.

[2] Y. Liu, W. Jiang, S. Jin, M. Rice, and Y. Chen, "Distributing power grid state estimation on HPC clusters - A system architecture prototype," in IEEE International Parallel and Distributed Processing Symposium Workshops, 2012, pp. 1467-1476.

[3] R. Ebrahimian and R. Baldick, "State estimation distributed processing [for power systems]," IEEE Trans. Power Syst., vol. 15, no. 4, pp. 1240$1246,2000$.

[4] L. Xie, D. H. Choi, S. Kar, and H. V. Poor, "Fully distributed state estimation for wide-area monitoring systems," IEEE Trans. Smart Grid, vol. 3, no. 3, pp. 1154-1169, 2012.

[5] V. Kekatos and G. B. Giannakis, "Distributed robust power system state estimation," IEEE Transactions on Power Systems, vol. 28, no. 2, pp. 1617-1626, 2013.

[6] A. Minot, Y. M. Lu, and N. Li, "A Distributed Gauss-Newton Method for Power System State Estimation," IEEE Trans. Power Systems, pp. $1-12,2015$.

[7] S. Yuan, P.-N. Tan, K. S. Cheruvelil, S. M. Collins, and P. A. Soranno, "Constrained spectral clustering for regionalization: Exploring the tradeoff between spatial contiguity and landscape homogeneity," in IEEE International Conference on Data Science and Advanced Analytics, October 2015, pp. 1-10.

[8] J. Shi and J. Malik, "Normalized cuts and image segmentation," IEEE Trans. Pattern Anal. Mach. Intell., vol. 22, no. 8, pp. 888-905, August 2000.

[9] U. von Luxburg, "A tutorial on spectral clustering," Statistics and Computing, vol. 17, no. 4, pp. 395-416, 2007.

[10] R. D. Christie, "Power systems test case archive." [Online]. Available: https://www.ee.washington.edu/research/pstca/ 